\title{
The Riemann Hypothesis Is Possibly True
}

\section{Frank Vega}

\begin{abstract}
In mathematics, the Riemann hypothesis is a conjecture that the Riemann zeta function has its zeros only at the negative even integers and complex numbers with real part $\frac{1}{2}$. The Riemann hypothesis belongs to the David Hilbert's list of 23 unsolved problems and it is one of the Clay Mathematics Institute's Millennium Prize Problems. The Robin criterion states that the Riemann hypothesis is true if and only if the inequality $\sigma(n)<e^{\gamma} \times n \times \log \log n$ holds for all natural numbers $n>5040$, where $\sigma(x)$ is the sum-of-divisors function and $\gamma \approx 0.57721$ is the Euler-Mascheroni constant. The Nicolas criterion states that the Riemann hypothesis is true if and only if the inequality $\prod_{q \leq q_{n}} \frac{q}{q-1}>e^{\gamma} \times \log \theta\left(q_{n}\right)$ is satisfied for all primes $q_{n}>2$, where $\theta(x)$ is the Chebyshev function. Using both inequalities, we show that the Riemann hypothesis is possibly true.
\end{abstract}

1. INTRODUCTION In mathematics, the Chebyshev function $\theta(x)$ is given by

$$
\theta(x)=\sum_{q \leq x} \log q
$$

where $q \leq x$ means all the prime numbers $q$ that are less than or equal to $x$. Let $N_{n}=2 \times 3 \times 5 \times 7 \times 11 \times \cdots \times q_{n}$ denotes a primorial number of order $n$ such that $q_{n}$ is the $n^{t h}$ prime number. Thus, $\theta\left(q_{n}\right)=\log N_{n}$. We define a sequence based on this function:

Definition. For every prime number $q_{n}$, we define the sequence of real numbers:

$$
X_{n}=\frac{\prod_{q \leq q_{n}} \frac{q+1}{q}}{\log \theta\left(q_{n}\right)} .
$$

We use this limit superior,

Theorem 1. [1].

$$
\limsup _{n \rightarrow \infty} X_{n}=\frac{e^{\gamma} \times 6}{\pi^{2}}
$$

Say Nicolas $\left(q_{n}\right)$ holds provided

$$
\prod_{q \leq q_{n}} \frac{q}{q-1}>e^{\gamma} \times \log \theta\left(q_{n}\right) .
$$

The constant $\gamma \approx 0.57721$ is the Euler-Mascheroni constant and $\log$ is the natural logarithm. The importance of this inequality is:

Theorem 2. Nicolas $\left(q_{n}\right)$ holds for all prime numbers $q_{n}>2$ if and only if the Riemann hypothesis is true [4].

Besides, we define the following properties of the Riemann zeta function, 
Theorem 3. [2].

$$
\prod_{k=1}^{\infty} \frac{q_{k}^{2}}{q_{k}^{2}-1}=\zeta(2)=\frac{\pi^{2}}{6} .
$$

Theorem 4. [2]. For $a \geq 1$ :

$$
\prod_{q}\left(1-\frac{1}{q^{a+1}}\right)=\frac{1}{\zeta(a+1)} .
$$

As usual $\sigma(n)$ is the sum-of-divisors function of $n[\mathbf{1}]$ :

$$
\sum_{d \mid n} d
$$

where $d \mid n$ means the integer $d$ divides $n$. Define $f(n)$ to be $\frac{\sigma(n)}{n}$. We know these properties for this function:

Theorem 5. [3]. Let $\prod_{i=1}^{m} q_{i}^{a_{i}}$ be the representation of $n$ as a product of primes $q_{1}<\cdots<q_{m}$ with natural numbers as exponents $a_{1}, \ldots, a_{m}$. Then,

$$
f(n)=\left(\prod_{i=1}^{m} \frac{q_{i}}{q_{i}-1}\right) \times \prod_{i=1}^{m}\left(1-\frac{1}{q_{i}^{a_{i}+1}}\right) .
$$

Theorem 6. [1]. For $n>1$ :

$$
f(n)<\prod_{q \mid n} \frac{q}{q-1}
$$

Say $\operatorname{Robins}(n)$ holds provided

$$
f(n)<e^{\gamma} \times \log \log n .
$$

The importance of this inequality is:

Theorem 7. Robins $(n)$ holds for all natural numbers $n>5040$ if and only if the Riemann hypothesis is true [5]. If the Riemann hypothesis is false, then there are infinitely many natural numbers $n>5040$ such that Robins $(n)$ does not hold [5].

It is known that $\operatorname{Robins}(n)$ holds for many classes of numbers $n$.

Theorem 8. Robins $(n)$ holds for all natural numbers $n>5040$ such that $n=N_{m}$, where $N_{m}$ is a primorial number of order $m[1]$.

Let $q_{1}=2, q_{2}=3, \ldots, q_{m}$ be the first $m$ consecutive primes, then an integer of the form $\prod_{i=1}^{m} q_{i}^{a_{i}}$ with $a_{1} \geq a_{2} \geq \cdots \geq a_{m} \geq 0$ is called an Hardy-Ramanujan integer [1]. Based on the theorem 7, we know this result:

Theorem 9. If the Riemann hypothesis is false, then there exist infinitely many natural numbers $n>5040$ which are an Hardy-Ramanujan integer and Robins $(n)$ does not hold [1]. 
2. ANCILLARY LEMMAS The following is a key lemma.

Lemma 1. There exists a natural number $N$ such that $X_{n}<\frac{e^{\gamma} \times 6}{\pi^{2}}+\varepsilon$ for all natural numbers $n>N$ and $\varepsilon<\frac{6}{\pi^{2}}$. Only a finite number of elements of the sequence are greater than $\frac{e^{\gamma} \times 6}{\pi^{2}}+\varepsilon$ (this could be an empty set).

Proof. The limit superior of a sequence of real numbers $y_{n}$ is the smallest real number $b$ such that, for any positive real number $\varepsilon$, there exists a natural number $N$ such that $y_{n}<b+\varepsilon$ for all natural numbers $n>N$. Only a finite number of elements of the sequence are greater than $b+\varepsilon$ (this could be an empty set). Therefore, this is a consequence of the theorem 1 .

Lemma 2. Let $\prod_{i=1}^{m} q_{i}^{a_{i}}$ be the representation of an Hardy-Ramanujan integer $n>$ 5040 as a product of the first $m$ primes $q_{1}<\cdots<q_{m}$ with natural numbers as exponents $a_{1} \geq a_{2} \geq \cdots \geq a_{m} \geq 0$. If Robins $(n)$ does not hold, then $\operatorname{Nicolas}\left(q_{m}\right)$ holds indeed.

Proof. When Robins $(n)$ does not hold, then

$$
f(n) \geq e^{\gamma} \times \log \log n .
$$

Let's assume that Nicolas $\left(q_{m}\right)$ does not hold as well. Consequently,

$$
\prod_{q \leq q_{m}} \frac{q}{q-1} \leq e^{\gamma} \times \log \log N_{m}
$$

According to the theorem 6,

$$
\begin{aligned}
e^{\gamma} \times \log \log N_{m} & \geq \prod_{q \leq q_{m}} \frac{q}{q-1} \\
& >f(n) \\
& \geq e^{\gamma} \times \log \log n .
\end{aligned}
$$

However, this implies that $N_{m}>n$ which is a contradiction since $n>5040$ is an Hardy-Ramanujan integer.

Lemma 3. If some prime number $q_{n}>2$ complies with

$$
X_{n} \leq \frac{e^{\gamma} \times 6}{\pi^{2}}
$$

then Nicolas $\left(q_{n}\right)$ does not hold.

Proof. If we have the inequality

$$
X_{n} \leq \frac{e^{\gamma} \times 6}{\pi^{2}}
$$

then this is equivalent to

$$
\prod_{q \leq q_{n}} \frac{q+1}{q} \leq \frac{e^{\gamma} \times 6}{\pi^{2}} \times \log \theta\left(q_{n}\right) .
$$

November 5, 2021]

THE RIEMANN HYPOTHESIS 
If we multiply the both sides by $\frac{\pi^{2}}{6}$, so

$$
\frac{\pi^{2}}{6} \times \prod_{q \leq q_{n}} \frac{q+1}{q} \leq e^{\gamma} \times \log \theta\left(q_{n}\right) .
$$

We use that theorem 3 to show that

$$
\frac{\pi^{2}}{6} \times \prod_{q \leq q_{n}} \frac{q+1}{q}>\left(\prod_{q \leq q_{n}} \frac{q^{2}}{q^{2}-1}\right) \times \prod_{q \leq q_{n}} \frac{q+1}{q} .
$$

Besides,

$$
\left(\prod_{q \leq q_{n}} \frac{q^{2}}{q^{2}-1}\right) \times \prod_{q \leq q_{n}} \frac{q+1}{q}=\prod_{q \leq q_{n}} \frac{q}{q-1}
$$

because of

$$
\frac{q}{q-1}=\frac{q^{2}}{q^{2}-1} \times \frac{q+1}{q} .
$$

Consequently, we obtain that

$$
\prod_{q \leq q_{n}} \frac{q}{q-1} \leq e^{\gamma} \times \log \theta\left(q_{n}\right)
$$

and therefore, $\operatorname{Nicolas}\left(q_{n}\right)$ does not hold.

\section{POSSIBLE PROOF OF THE RIEMANN HYPOTHESIS}

Theorem 10. The Riemann hypothesis is possibly true.

Proof. Let $\prod_{i=1}^{m} q_{i}^{a_{i}}$ be the representation of a sufficiently large Hardy-Ramanujan integer $n>5040$ as a product of the first $m$ primes $q_{1}<\cdots<q_{m}$ with natural numbers as exponents $a_{1} \geq a_{2} \geq \cdots \geq a_{m} \geq 0$. We claim that for every sufficiently large Hardy-Ramanujan integer $n>5040$, then Robins $(n)$ could always hold. Suppose that Robins $(n)$ does not hold and so, the Riemann hypothesis would be false. Hence,

$$
f(n) \geq e^{\gamma} \times \log \log n .
$$

We use that theorem 5,

$$
\left(\prod_{i=1}^{m} \frac{q_{i}}{q_{i}-1}\right) \times \prod_{i=1}^{m}\left(1-\frac{1}{q_{i}^{a_{i}+1}}\right) \geq e^{\gamma} \times \log \log n
$$

which is equivalent to

$$
\left(\prod_{i=1}^{m} \frac{q_{i}^{2}}{q_{i}^{2}-1}\right) \times\left(\prod_{i=1}^{m} \frac{q_{i}+1}{q_{i}}\right) \times \prod_{i=1}^{m}\left(1-\frac{1}{q_{i}^{a_{i}+1}}\right) \geq e^{\gamma} \times \log \log n .
$$


If we divide the both sides by $\log \log N_{m}$, then we obtain

$$
\left(\prod_{i=1}^{m} \frac{q_{i}^{2}}{q_{i}^{2}-1}\right) \times X_{m} \times \prod_{i=1}^{m}\left(1-\frac{1}{q_{i}^{a_{i}+1}}\right) \geq e^{\gamma} \times \frac{\log \log n}{\log \log N_{m}}
$$

because of $\log \log N_{m}=\log \theta\left(q_{m}\right)$, where $N_{m}$ is the primorial number of order $m$. We know that $X_{m} \leq \frac{e^{\gamma} \times 6}{\pi^{2}}$ is false according to the lemmas 2 and 3. From the lemma 1, we know that there exists a natural number $N$ such that $X_{m}<\frac{e^{\gamma} \times 6}{\pi^{2}}+\varepsilon$ for all natural numbers $m>N$ and $\varepsilon<\frac{6}{\pi^{2}}$. Moreover, only a finite number of elements of the sequence are greater than $\frac{e^{\gamma} \times 6}{\pi^{2}}+\varepsilon$ (this could be an empty set). Under our assumption, there exist infinitely many Hardy-Ramanujan integers $n>5040$ such that Robins $(n)$ does not hold and $X_{m}<\frac{e^{\gamma} \times 6}{\pi^{2}}+\varepsilon$. In this way, we obtain that

$$
\left(\prod_{i=1}^{m} \frac{q_{i}^{2}}{q_{i}^{2}-1}\right) \times\left(\frac{e^{\gamma} \times 6}{\pi^{2}}+\varepsilon\right) \times \prod_{i=1}^{m}\left(1-\frac{1}{q_{i}^{a_{i}+1}}\right) \geq e^{\gamma} \times \frac{\log \log n}{\log \log N_{m}}
$$

which is the same as

$$
\left(\prod_{i=1}^{m} \frac{q_{i}^{2}}{q_{i}^{2}-1}\right) \times \frac{6}{\pi^{2}} \times\left(e^{\gamma}+c\right) \times \prod_{i=1}^{m}\left(1-\frac{1}{q_{i}^{a_{i}+1}}\right) \geq e^{\gamma} \times \frac{\log \log n}{\log \log N_{m}}
$$

for a sufficiently small constant $c=\varepsilon \times \frac{\pi^{2}}{6}$. That would be equivalent to

$$
\left(\prod_{q>q_{m}} \frac{q^{2}-1}{q^{2}}\right) \times\left(e^{\gamma}+c\right) \times \prod_{i=1}^{m}\left(1-\frac{1}{q_{i}^{a_{i}+1}}\right) \geq e^{\gamma} \times \frac{\log \log n}{\log \log N_{m}} .
$$

Since $n$ is an Hardy-Ramanujan integer, then

$$
\left(\prod_{q>q_{m}} \frac{q^{2}-1}{q^{2}}\right) \times \prod_{i=1}^{m}\left(1-\frac{1}{q_{i}^{a_{i}+1}}\right)<\prod_{q}\left(1-\frac{1}{q^{a_{1}+1}}\right)=\frac{1}{\zeta\left(a_{1}+1\right)}
$$

because of the theorem 4 , where $a_{1}$ is the highest exponent such that $2^{a_{1}} \mid n$. Therefore,

$$
\frac{\left(e^{\gamma}+c\right)}{\zeta\left(a_{1}+1\right)}>e^{\gamma} \times \frac{\log \log n}{\log \log N_{m}}
$$

for a sufficiently small constant $0<c<1$. However, this could be false for a sufficiently small value of $\varepsilon<\frac{6}{\pi^{2}}$ that we could choose, where $c=\varepsilon \times \frac{\pi^{2}}{6}$ would be a very small constant as well. In addition, we know that $\frac{\log \log n}{\log \log N_{m}}>1$ due to the theorem 8 . In conclusion, for every sufficiently large Hardy-Ramanujan integer $n>5040$, then Robins $(n)$ could always hold. By contraposition, the Riemann hypothesis is possibly true, because of the theorems 7 and 9 .

ACKNOWLEDGMENT. The author wishes to thank Richard J. Lipton and Craig Helfgott for helpful comments and his mother, maternal brother and his friend Sonia for their support. 


\section{REFERENCES}

1. Choie, Y., Lichiardopol, N., Moree, P., Solé, P. (2007). On Robin's criterion for the Riemann hypothesis. Journal de Théorie des Nombres de Bordeaux, 19(2): 357-372. http://dx.doi .org/10.5802/jtnb. 591.

2. Edwards, H. M. (2001). Riemann's Zeta Function. Dover Publications.

3. Hertlein, A. (2018). Robin's Inequality for New Families of Integers. Integers, 18.

4. Nicolas, J.-L. (1983). Petites valeurs de la fonction d'Euler. Journal of number theory, 17(3): 375-388. http://dx.doi.org/10.1016/0022-314X (83) 90055-0.

5. Robin, G. (1984). Grandes valeurs de la fonction somme des diviseurs et hypothèse de Riemann. J. Math. pures appl, 63(2): 187-213.

FRANK VEGA is essentially a back-end programmer who graduated in Computer Science in 2007. In August 2017, he was invited as a guest reviewer for a blind peer-review of a manuscript about Theory of Computation in the flagship journal of IEEE Computer Society. In October 2017, he contributed as co-author with a presentation about economy in the 7th International Scientific Conference on economic development and standard of living ("EDASOL 2017 - Economic development and Standard of living"). In June 2018, this paper was published by the Journal of Economics and Market Communications (EMC). In February 2017, his book "Protesta" (a book of poetry and short stories in Spanish) was published by the Alexandria Library Publishing House. In September 2019, a scientific paper entitled "Triangle Finding" was accepted by the conference IMCS-55. He was Director of two IT Companies (Joysonic and Chavanasoft) created in Serbia.

CopSonic, 1471 Route de Saint-Nauphary 82000 Montauban, France

vega.frank@gmail.com 